Conclusions The costs of an Advance Care Planning programme were limited. Advance Care Planning did not significantly affect the costs of medical care for frail older adults.

\section{OP58 NEW US ADVANCE CARE PLANNING BILLING CODES - WHO'S USING IT AND FOR WHOM?}

${ }^{1} \mathrm{H}$ Lum*, ${ }^{2} \mathrm{P}$ Shanbhag, ${ }^{2} \mathrm{~A}$ Daddato. ${ }^{1}$ University of Colorado and VA GRECC, Aurora, USA; ${ }^{2}$ University of Colorado School Of Medicine, Aurora, USA

\subsection{6/spcare-2019-ACPICONGRESSABS.58}

Background In the US, new reimbursement for advance care planning (ACP) started on January 1, 2016. This study aims to describe patient and practitioner characteristics related to use of the new billing codes, including documentation of the ACP process and advance directives.

Methods Retrospective, cross-sectional analysis of the billing code 99497 from January 1, 2016 thru June 30, 2018 in outpatient visits in a large healthcare system. We describe patientlevel and practitioner-level characteristics. We reviewed clinical documentation elements from a sample of patient visits from high- and low-utilizing practitioners.

Results Seventy-six practitioners used the ACP billing code in 3421 outpatient visits for 2884 patients. Patients were mean age 73 (range 20-104 years), 57\% female, and 2\% rural residing. $35 \%$ of patients had an advance directive on file. Mean number of billing encounters per practitioner was 45 (range 1-704). Visits occurred in primary care settings (family medicine, internal medicine, geriatric medicine) and two subspecialty clinics (neurology, cardiology). ACP was billed multiple times for 150 patients (5.2\%), with a range of two to four visits. The average time between unique visits was 330 days. The most commonly documented topics were code status, POLST/MOST form, and surrogate decision maker. 28\% of ACP documents on file were completed within seven days of the ACP billing visit.

Conclusions This is the first study to describe use of ACP billing codes in outpatient settings. Practitioners are using the ACP billing code mostly in primary care settings, among older adults, and occasionally multiple times.

\section{OP59 COMPLEX INTERVENTIONS GUIDING ADVANCE CARE PLANNING CONVERSATIONS: A SYSTEMATIC REVIEW}

${ }^{1} \mathrm{~J}$ Fahner*, ${ }^{1} \mathrm{~A}$ Beunders, ${ }^{1} \mathrm{~J}$ van Delden, ${ }^{2} \mathrm{~A}$ Van der Heide, ${ }^{1} \mathrm{M}$ Vanderschuren, ${ }^{2} \mathrm{~J}$ Rietjens, ${ }^{1} \mathrm{M}$ Kars. 'University Medical Center Utrecht, Utrecht, Netherlands; ${ }^{2}$ Erasmus MC, Rotterdam, Netherlands

\subsection{6/spcare-2019-ACPICONGRESSABS.59}

Background Conversation guides support professionals to conduct ACP-conversations, yet insight in essential components is limited. This systematic review aims to evaluate the content, rationale and empirical evidence on the effect of ACP interventions based on conversation guides.

Methods Medline, Embase, PsychINFO and CINAHL were searched from January 1, 1998 to February 23, 2018 to identify peer-reviewed articles describing or evaluating scripted ACP-conversation guides. A thematic analysis of the guides was performed. Data on intervention characteristics, underlying rationale and empirical evidence was extracted.
Results Eighty-two articles reporting on thirty-four unique interventions met the inclusion criteria. Analysis of the conversation guides revealed a framework for ACP-conversations consisting of four phases: preparation, initiation, exploration and action. Exploration of patient's perspectives on illness, living well, end-of-life (EOL) issues and decision making formed the core part of the guides. Their design was often expert-based, without an underlying theoretical background. Empirical evidence on the effect of the interventions was based on heterogeneous outcome measures. Dyad congruence and preference documentation rates increased among intervention subjects in most studies. The studies showed varying effects on knowledge of ACP, decisional conflict, quality of communication and preferences-concordant care. Qualitative research showed that participants appreciate the importance and benefits of ACP-conversations, yet perceive them as difficult and emotional.

Conclusion ACP-conversation guides address a diversity of themes regarding illness, EOL and decision making, with a focus on the exploration of patient's perspectives and preferences. Evidence on translation of explorative information into specific treatment preferences and consequences for care as provided is limited.

\section{OP60 EVALUATION AND FURTHER DEVELOPMENT OF A DUTCH QUESTION PROMPT LIST ON PALLIATIVE CARE FROM THE PERSPECTIVE OF PATIENTS AND FAMILY}

DWH Vergroesen*, M Verhoef, N Horeweg, AH Pieterse, EJM De Nijs, YM van der Linden. Leiden University Medical Centre, Leiden, Netherlands

\subsection{6/spcare-2019-ACPICONGRESSABS.60}

Background Patients and family often do not know what to expect of an advance care planning (ACP) consultation. Question prompt lists (QPL) help them to gain insight into and express their wishes and questions. We developed the 'Leiden Guide for Palliative care' (LGP), combining an adapted Edmonton Symptom Assessment Scale and a translated QPL on palliative care, ${ }^{1}$ to hand out before the ACP consultation with palliative care specialists. The goals of this study were to evaluate personal experiences of patients and family with the LGP, and to further develop the LGP.

Methods In this qualitative study semi-structured interviews with six patients and seven family members were conducted. Manual coding and thematic analysis were done by two researchers.

Results Three main themes for optimal use of the LGP were identified: 1. Prerequisites: early in disease trajectory; adequate introduction by the healthcare professional (HCP); positive first impression. 2. Benefits: provides complete overview of ACP topics and relevant questions; facilitates end of life discussions, also between family members. 3. Practical use: preferably the LGP is reviewed with family 1-2 days before the consultation. With detailed suggestions on content and format we constructed an improved LGP.

Conclusion Patients and family consider the LGP as helpful and useful in preparation and during ACP consultations with palliative care specialists, provided that the prerequisites are met. The usefulness of the LGP in ACP discussions with generalist HCPs and in different settings is subject of further study.

\section{REFERENCE}

1. Clayton J, et al. Br J Cancer 2003. 